Original Article

\title{
Berberine alleviates symptoms of anxiety by enhancing dopamine expression in rats with post-traumatic stress disorder
}

\author{
Bombi Lee ${ }^{1,2 * *}$, Insop Shim ${ }^{3}$, Hyejung Lee ${ }^{1}$, and Dae-Hyun Hahm ${ }^{3, *}$ \\ ${ }^{1}$ Acupuncture and Meridian Science Research Center, College of Korean Medicine, ${ }^{2}$ Center for Converging Humanities, ${ }^{3}$ Department of Physiology, College of \\ Medicine, Kyung Hee University, Seoul 02447, Korea
}

\section{ARTICLE INFO}

Received October 13, 2017

Revised December 22, 2017

Accepted December 28, 2017

*Correspondence

Bombi Lee

E-mail: bombi@khu.ac.kr

Dae-Hyun Hahm

E-mail: dhhahm@khu.ac.kr

Key Words

Anxiety

Berberine

Dopamine

Post-traumatic stress disorder

Single prolonged stress
ABSTRACT Post-traumatic stress disorder (PTSD) is a trauma-induced psychiatric disorder characterized by impaired fear extermination, hyperarousal, anxiety, depression, and amnesic symptoms that may involve the release of monoamines in the fear circuit. The present study measured several anxiety-related behavioral responses to examine the effects of berberine (BER) on symptoms of anxiety in rats after single prolonged stress (SPS) exposure, and to determine if BER reversed the dopamine (DA) dysfunction. Rats received BER (10,20, or $30 \mathrm{mg} / \mathrm{kg}$, intraperitoneally, once daily) for 14 days after SPS exposure. BER administration significantly increased the time spent in the open arms and reduced grooming behavior during the elevated plus maze test, and increased the time spent in the central zone and the number of central zone crossings in the open field test. BER restored neurochemical abnormalities and the SPS-induced decrease in DA tissue levels in the hippocampus and striatum. The increased DA concentration during BER treatment may partly be attributed to mRNA expression of tyrosine hydroxylase and the DA transporter in the hippocampus, while BER exerted no significant effects on vesicular monoamine transporter mRNA expression in the hippocampus of rats with PTSD. These results suggest that BER had anxiolytic-like effects on behavioral and biochemical measures associated with anxiety. These findings support a role for reduced anxiety altered DAergic transmission and reduced anxiety in rats with PTSD. Thus, BER may be a useful agent to treat or alleviate psychiatric disorders like those observed in patients with PTSD.

\section{INTRODUCTION}

Post-traumatic stress disorder (PTSD) is a stress-induced psychiatric disorder that is associated with marked deficiencies in social functioning [1]. PTSD can develop in response to a traumatic, life-threatening event [2]. The characteristic signs of PTSD include hyperarousal, insensibility, fear, and nightmares, leading to high rates of comorbidity with psychosocial impairment and anxiety $[3,4]$. The onset of PTSD often precedes and increases the risk for subsequent development of anxiety disorders, and PTSD enhances the susceptibility to anxiety [5]. Although the neurobiology of PTSD is not fully understood, preclinical and clinical studies have implicated altered dopamine (DA) activity in the disorder [6].

It is widely accepted that neuronal system of catecholamine dysfunction in general and DA in particular play an important role in the pathophysiology of PTSD [1]. DAergic neurotransmission in the mesolimbic pathway is important for mediating arousal and memory, and altered DA activity may contribute to hyperarousal and re-experiencing symptoms associated with PTSD [2]. Central DA appears to positively affect fear conditioning [7]. For example, fear extinction is postponed in rats with restrained prefrontal or hippocampal DA function, indicating that DA is particularly crucial within the cortex [7]. (i) \$ This is an Open Access article distributed under the terms of the Creative Commons Attribution Non-Commercial License, which permits unrestricted non-commercial use, distribution, and reproduction in any medium, provided the original work is properly cited. Copyright @ Korean J Physiol Pharmacol, pISSN 1226-4512, elSSN 2093-3827
Author contributions: B.L. and D.H.H. performed the conception and design. B.L. performed the carried out the experiments. B.L. and D.H.H. performed the acquisition of data. B.L. and D.H.H. performed the analysis and interpretation. B.L. performed the drafting the article. B.L. and D.H.H. performed the statistical analysis. I.S. and H.L. performed the study supervision. 
Several rodent models have been used to investigate the fear mechanism of PTSD. The single prolonged stress (SPS) model, which simulates a traumatic stressful event, has been extensively employed because it yields high validity when examining traumainduced learning or anxiety $[8,9]$. Rats exposed to SPS show enhanced dysregulation of the hypothalamic-pituitary-adrenal (HPA) axis [10,11], increased anxiety-like behavior on the elevated plus maze (EPM) test, and increased fear conditioning [9]. These responses imitate the clinical symptoms observed in patients with PTSD [12]. Many studies have shown that dysregulation in brain DA circuitry can lead to the pathological state of PTSD and produce anxiety-like symptoms [13].

Fluoxetine (FLX) is an antidepressant known to be effective for treating patients with PTSD [14]. Some studies have shown that FLX increases synaptic plasticity and fear extinction via the serotonergic signaling pathway, which alters DA and serotonin levels in the brain [15]. Although selective serotonin reuptake inhibitors, including FLX, are efficacious in many patients, they also have deleterious effects that limit their use, including psychiatric symptoms, including loss of weight, sedation, and sexual dysfunction [16]. Therefore, there is an ongoing need for promising PTSD treatments [17]. Consequently, many studies have explored the use of natural medicines that may be safer for long-term therapy [18].

In the present study, we explored the pharmacological activity of berberine (BER, $\mathrm{C}_{20} \mathrm{H}_{18} \mathrm{CINO}_{4}$ ), an isoquinoline alkaloid derived from Korean traditional medicinal herbs, such as Berberis, Hydrastis canadensis, Rhizoma coptidis, and Cortex phellodendri [19]. BER is widely reported to improve multiple physiological actions and produces a variety of biological effects in the central nervous system (CNS) [20,21]. BER exerts neuroprotective, antioxidant, anti-apoptotic, antitumor, antiviral, and anti-inflammatory properties in various animal models of CNSrelated disorders, such as Alzheimer's disease, Parkinson's disease (PD), forebrain ischemia, depression, and anxiety [19,22-24]. BER inhibited 6-hydroxydopamine-induced neurotoxicity medicated by the formation of reactive oxygen species in PC12 cells and in an animal model of PD [22]. The pharmacological profile of BER, which includes high-affinity binding to DA receptors, suggests that it may be useful for treating drug addiction, such as cocaine and morphine [25]. Specifically, BER inhibits DA biosynthesis and reduces DA content in PC12 cells [22]. Therefore, BER appears to contribute to many of the therapeutic effects of these preparations, presumably through its interactions with the DA receptor [26]. BER also significantly reduces the total duration of immobility on the forced swimming test and tail-suspension test [19], and ameliorates anxiety-related behavior by activating the serotonergic system in mice [27]. Therefore, the present study hypothesized that BER would be protective against the development of anxiety-like behaviors, which represent the core symptoms of PTSD-related abnormalities, in a rat model of PTSD using SPS. Identifying the neuropathology of the mesolimbic DA system may help in understanding the functional relationship between DA and PTSD. DA dysfunction may also be relevant to the mechanisms underlying emotional numbing, a symptom of PTSD.

Although a brief report was published on the antidepressant and anti-stress effects of BER [19,21], questions remain regarding the mechanisms underlying the effect of BER as an alternative therapeutic intervention for treating anxiety-like symptoms following SPS exposure in rats. The present study investigated the medicinal effects of BER on anxiety-related behaviors in rats exposed to SPS using the open field test (OFT) and the EPM test, representing the core symptoms of PTSD-related abnormalities. Moreover, we examined how the behavioral effects were associated with the DAergic system in the brain as an underlying mechanism.

\section{METHODS}

\section{Animals and BER administration}

Six-week-old adult male Sprague-Dawley rats (Samtako Animal Co., Seoul, Korea), weighing 210-240 g, were used in all experiments. The rats were housed under a 12-h light/dark cycle (lights on at 8:00 am, lights off at 8:00 pm) under a controlled temperature at $23 \pm 5^{\circ} \mathrm{C}$ and relative humidity of $50 \pm 10 \%$. All rats were allowed to adapt to these conditions for 7 days after they arrived. All methods and procedures were approved by the Animal Care and Use Committee of Kyung Hee University [KHUASP(SE)-15-115]. All procedures were executed according to the Guide for the Care and Use of Laboratory Animals.

BER $(10,20$, and $30 \mathrm{mg} / \mathrm{kg}$, body weight; Sigma-Aldrich Chemical Co., St. Louis, MO, USA) and the positive drug FLX for positive control group $(10 \mathrm{mg} / \mathrm{kg}$, fluoxetine hydrochloride; Sigma) were injected by intraperitoneally (i.p.) after exposure to SPS. Six or seven animals per group were used. BER and FLX were dissolved in $0.9 \%$ physiological saline before use. The entire experimental schedules of all drug administration and behavioral examinations are shown in Fig. 1.

\section{Single prolonged stress}

Rats were subjected to SPS for 14 consecutive days as described by Patki's group, with a slight modification [14,28]. Briefly, rats were held for $2 \mathrm{~h}$ in Plexiglas cylinders, then promptly entered a forced swimming condition for $20 \mathrm{~min}$. The rats were dried and allowed to recuperate for $15 \mathrm{~min}$, and then were exposed to ether vapor until they lost consciousness. Following the SPS stressor, the rats were housed one per cage and left undisturbed for 14 days to allow PTSD-like symptoms to manifest [14]. 


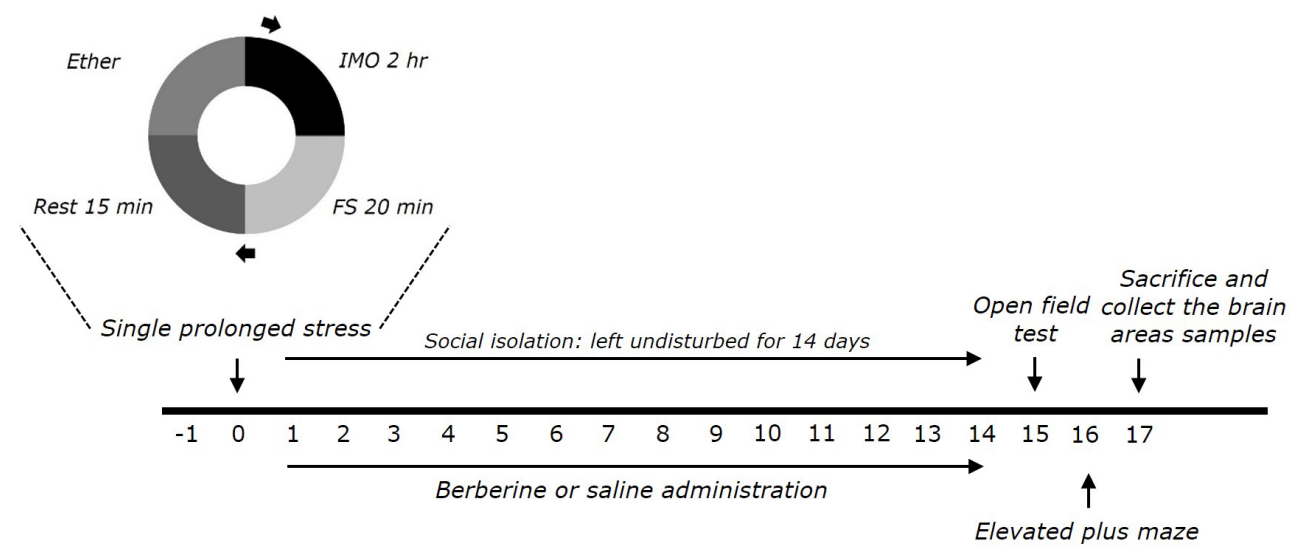

Fig. 1. Experimental schedule for developing single prolonged stress (SPS)-induced anxiety-like behaviors, and berberine (BER) treatment in rats. Separate groups of rats ( $n=6$ or 7 animals per group) were used for all experiments.

\section{Elevated plus maze (EPM) test}

The EPM test was carried out according to a method described previously [29]. Briefly, the rats were transferred to the EPM, which consisted of a 4 -armed wooden platform in the shape of a plus sign. The apparatus was painted with black enamel and was raised $50 \mathrm{~cm}$ above the floor. All arms were $50 \mathrm{~cm}$ in length, 10 $\mathrm{cm}$ in width, and joined in the center to create a $10 \mathrm{~cm}^{2}$ center platform. Two arms facing away from each other were protected, whereas the remaining two arms remained open. At the start of each experiment, the rat could move freely for $5 \mathrm{~min}$. The video footage of these sessions was scored. The ratio of the total time spent in the open arms was used to measure anxiety.

\section{Open field test (OFT)}

Before completing the EPM test, the rats were subjected to the OFT. The OFT was carried out according to a method described previously [14]. Each rat was housed individually in a rectangular container $(60 \times 60 \times 30 \mathrm{~cm})$ in a dimly lit room. This provided the best contrast for white rats in a dimly lit room equipped with a video camera above the center of the room. Locomotor activities were indicated by the speed and distance of movements and monitored by a computerized video-tracking system using the SMART program (PanLab Co., Barcelona, Spain). Painted white lines divided the area into 16 squares $(15 \times 15 \mathrm{~cm}$ each). Each rat could freely explore the arena for $5 \mathrm{~min}$. Locomotion (central zone crossing) and time spent in the central and peripheral zones were observed. The number of rearing events for each rat was also recorded to analyze locomotor activity in the OFT.

\section{DA measurement}

Fourteen days after inducing PTSD, DA concentration was assayed in brain tissue using a method described previously [14]. Four rats from each group were deeply anesthetized with isoflurane (1.2\%), and were killed by sacrifice one day after the behavioral testing. The medial prefrontal cortex, hippocampus, striatum, and amygdala were quickly dissected from the rat brains in random order. The DA concentration was assessed by a competitive enzyme-linked immunoassay (ELISA) using a mouse monoclonal DA antibody (DA ELISA Kit; Abcam, Cambridge, MA, USA).

\section{Total RNA isolation and reverse transcription- polymerase chain reaction (RT-PCR)}

The levels of tyrosine hydroxylase (TH), DA transporter (DAT), and vesicular monoamine transporter-2 (VMAT-2) mRNA expression were measured by RT-PCR according to a method described previously [30]. In brief, total RNA was isolated from the hippocampus of each rat using TRIzol reagent according to the manufacturer's instructions. cDNA was synthesized from $2 \mu \mathrm{g}$ total RNA using reverse transcriptase (Takara Bio, Otsu, Japan), and then amplified by PCR at $60^{\circ} \mathrm{C}$ for 30 cycles for $\mathrm{TH}$, at $60^{\circ} \mathrm{C}$ for 30 cycles for DAT, and at $56^{\circ} \mathrm{C}$ for 35 cycles for VMAT-2 using Taq DNA polymerase (Takara, Kyoto, Japan) on a thermal cycler (MJ Research, Watertown, MA, USA). Data were normalized against glyceraldehyde 3-phosphate dehydrogenase (GADPH) expression in the corresponding sample.

\section{Statistical analysis}

Results are expressed as mean \pm standard error. Differences within or between normally distributed data were analyzed using an analysis of variance (ANOVA) with SPSS (version 13.0; SPSS, Inc., Chicago, IL, USA) and Tukey's post hoc tests. A p-value $<0.05$ was considered significant. 


\section{RESULTS}

\section{Effects of BER on anxiety-like behaviors following SPS}

Rats exhibited an obvious anxiety phenotype characterized by decreased open-arm exploration during the EPM test. Statistical analyses of the behavioral results showed that the percentage of time spent in the open arms of the maze significantly differed among the 6 groups $[\mathrm{F}(5,35)=3.397, \mathrm{p}<0.05]$. The ANOVA also revealed a significant effect of the number of open arm entries among the six groups $[\mathrm{F}(5,35)=6.540, \mathrm{p}<0.01]$. Post-hoc comparisons indicated that the PTSD groups showed a significantly decrease in both the percentage of time spent and the number of entries in the open arms of the maze than control group $(\mathrm{p}<0.01)$.
However, rats in the PTSD+BER30 group showed significant restoration of the time spent in the open arms of the maze compared with that of the PTSD group $(\mathrm{p}<0.05$; Fig. 2). Also, rats in the PTSD+BER30 group showed restoration of the number of entries in the open arms of the maze compared with that of the PTSD group, although this result was only marginally significant. The time and number of enclosed-arm entries did not differ significantly among the six groups $[\mathrm{F}(5,35)=4.988, \mathrm{p}=0.012$ and $\mathrm{F}(5.35)=2.051, \mathrm{p}=0.100]$. BER administration after SPS elicited anxiogenic or anxiolytic behavior. These findings indicate that the increased time spent in the open arms of the maze in the PTSD+BER30 group was comparable to the exploratory behavior in the PTSD+FLX group. Overall, the anxiety index, calculated based on the number of visits to and time spent in the open and
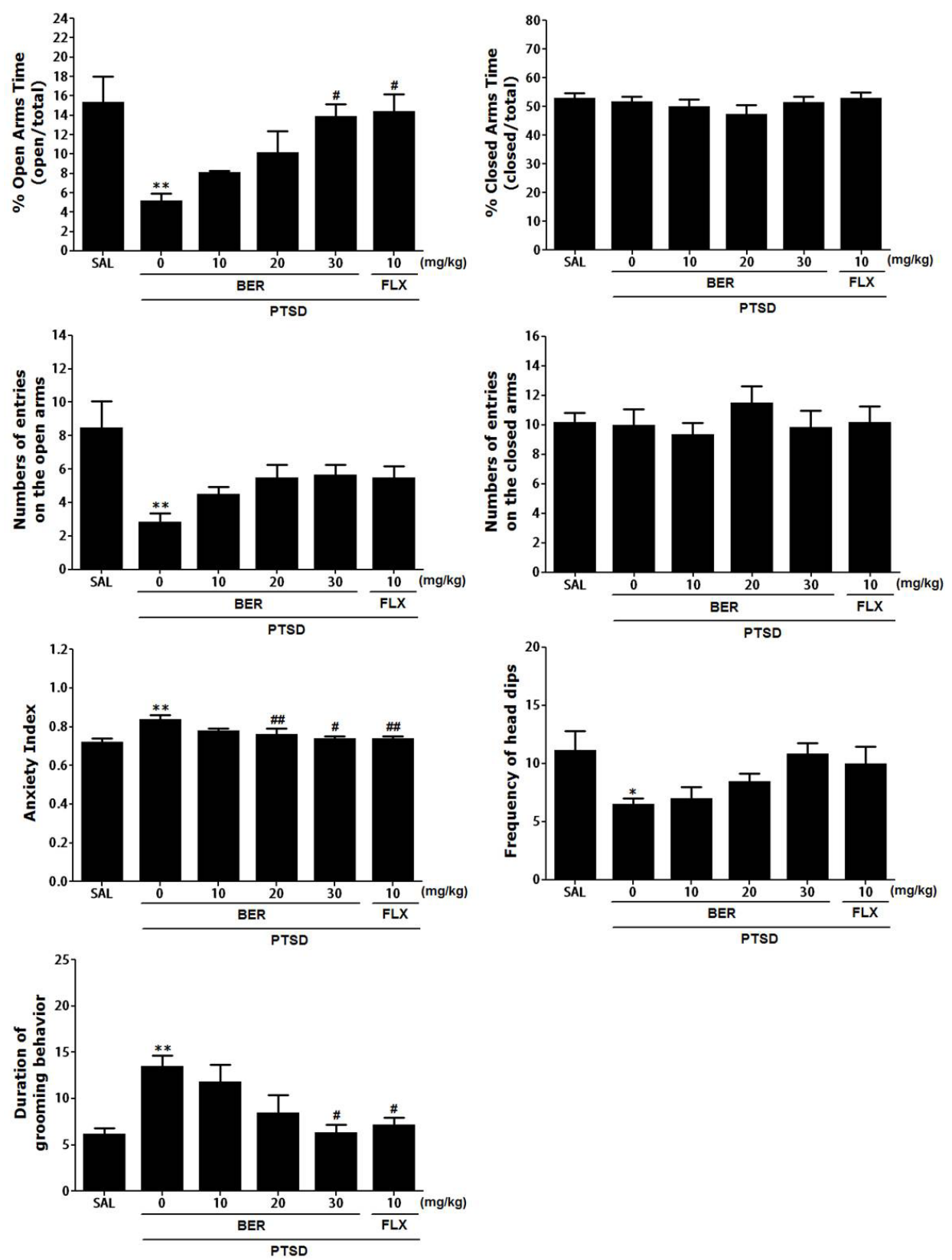

Fig. 2. Effects of BER administration on the percentage of time spent in the open and closed arms, numbers of entries into the open and closed arms, anxiety index, unprotected head dips, and grooming behavior on the elevated plus maze (EPM) test of rats exposed to SPS. ${ }^{*} p<0.05,{ }^{* *} p<0.01$ vs. SAL group; ${ }^{p} \mathrm{p}<0.05,{ }^{\#} \mathrm{p}<0.01$ vs. PTSD group.

https://doi.org/10.4196/kjpp.2018.22.2.183 
closed arms, also differed among the six groups of rats with lower values in the BER-treated rats $(\mathrm{p}<0.01$ or $\mathrm{p}<0.05)$. Administering BER significantly decreased the frequency of unprotected head dips compared with that in the PTSD group, although this result was only marginally significant. However, the duration of grooming behavior was reversed by $30 \mathrm{mg} / \mathrm{kg}$ BER when administered after SPS exposure $(\mathrm{p}<0.05)$.

\section{Effects of BER on locomotion and exploratory behavior following SPS}

Open-field activity was used to assess exploratory behavior and locomotion among rats exposed to SPS for 14 days (Fig. 3). Rats exposed to SPS had a significantly decreased amount of time spent in the central zone, with a corresponding increase in time spent in the peripheral zone, compared with the saline (SAL) group $(\mathrm{p}<0.01)$. A significant reduction in the number of central zone crossings was observed following the SPS procedure $(\mathrm{p}<0.01)$. Our results indicate that SPS-treated rats developed exploratory activities that were closely associated with the anxiety-like behaviors observed in the OFT. However, the BER-treated rats (30 mg/ $\mathrm{kg}$ ) exhibited a significant increase in the number of central zone crossings compared with that in the PTSD group $(\mathrm{p}<0.05)$, indicating that the anxiety-like behaviors of the PTSD+BER30 group were similar to those of the PTSD+FLX group. In addition, the BER-treated rats $(30 \mathrm{mg} / \mathrm{kg}$ ) exhibited a significant increase in the time spent in the central zone compared with that in the PTSD group ( $\mathrm{p}<0.01)$, indicating that the anxiety-like behaviors of the PTSD+BER30 group were similar to those of the PTSD+FLX group. A one-way ANOVA was performed, and PTSD-related differences were discovered in locomotor activity (motor func- tion) and the total number of rearing (exploratory activities) in the OFT (Fig. 3). A significant difference in locomotor activity was observed between saline-treated rats, the PTSD group, and the BER-treated groups $[\mathrm{F}(5,35)=3.363, \mathrm{p}<0.05]$ and the total times reared $[\mathrm{F}(5,35)=9.568, \mathrm{p}<0.001]$. Rats exposed to SPS had significantly decreased moving distance and rearing frequency in the open field compared with the SAL group ( $\mathrm{p}<0.05$ and $\mathrm{p}<0.01$ ). However, rats in the PTSD+BER30 group showed a significant restoration of the moving distance and rearing frequency in the open field compared with that in the PTSD group $(\mathrm{p}<0.05$ and $\mathrm{p}<0.01)$.

\section{Effects of BER on DA concentration in the hippocampus following SPS}

Fig. 4 shows that the brain region levels of DA were significantly different when the group were compared. Tissue levels of DA were measured in the medial prefrontal cortex, hippocampus, striatum, and amygdala after 2 week later (Day 14). The post-hoc test results indicated a significant decrease in the levels of DA in the hippocampus of the PTSD groups compared with that in the untreated PTSD group $(\mathrm{p}<0.05)$. Daily administration of BER significantly inhibited the SPS-induced decrease in DA concentration in the hippocampus compared with that in the PTSD group $(\mathrm{p}<0.05)$. After BER treatment, the levels of DA in the striatum also increased significantly to $256.80 \%$ of that in the PTSD group $(\mathrm{p}<0.05)$. The DA concentrations in the brain regions of rats receiving $10 \mathrm{mg} / \mathrm{kg}$ FLX were similar to those in rats receiving 30 $\mathrm{mg} / \mathrm{kg}$ BER.

The ELISA showed that SPS exposure for 14 days significantly decreased the DA concentration in the medial prefrontal cortex
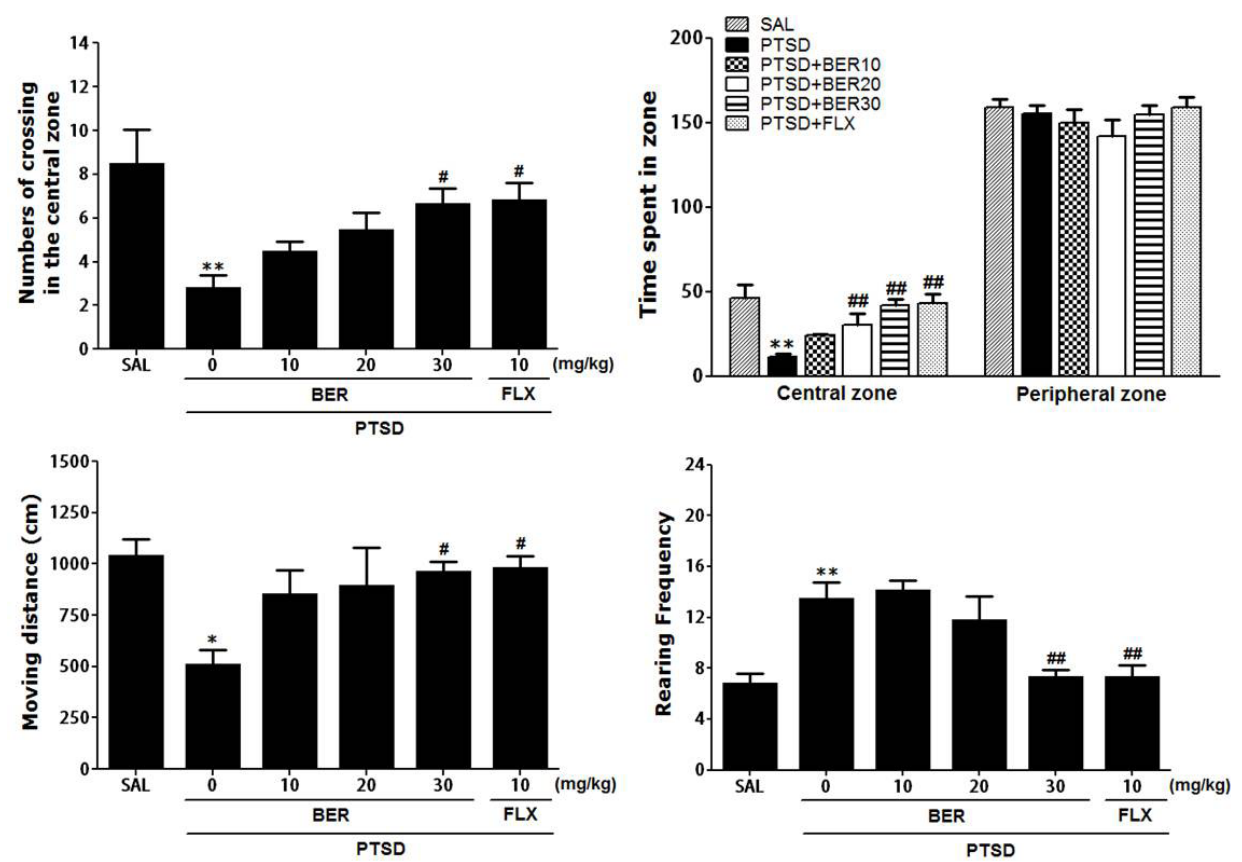

Fig. 3. Effects of BER administration on locomotion and exploratory behavior in the open filed test (OFT) of rats exposed to SPS. Change in the number of crossing in the central zone and the time spent in the central and peripheral zones. ${ }^{*} p<0.05,{ }^{* *} p<0.01$ vs. SAL group; ${ }^{\#} \mathrm{p}<0.05,{ }^{\# \#} \mathrm{p}<0.01$ vs. PTSD group. 
Medical prefrontal cortex

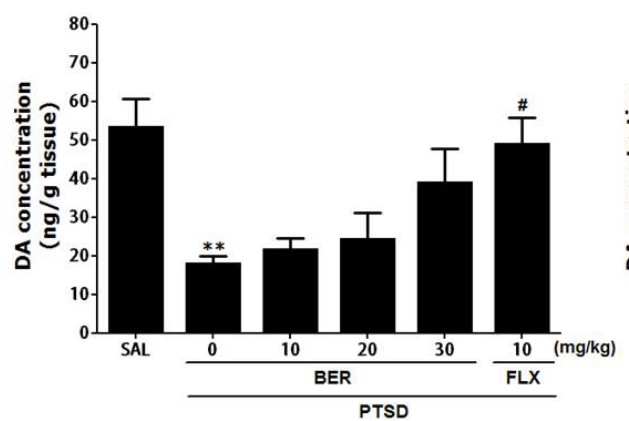

Hippocampus
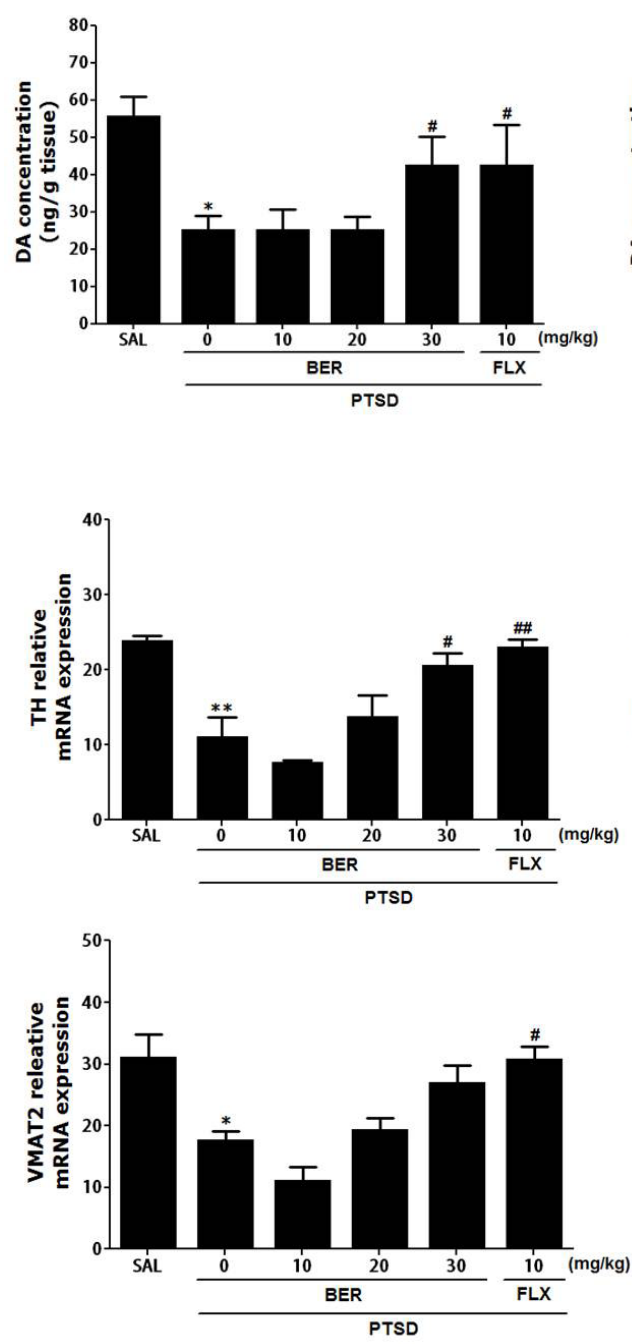

\section{Striatum}

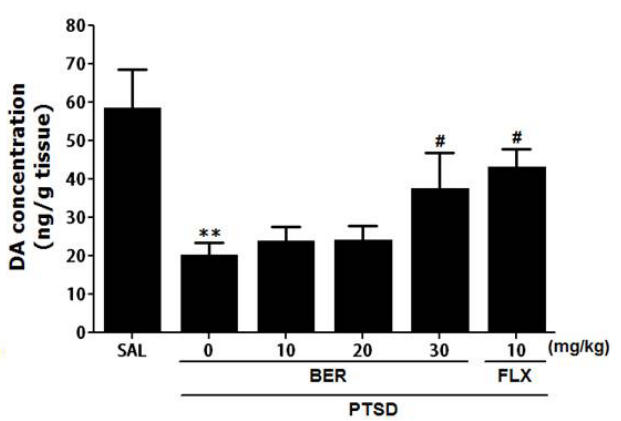

Amygdala
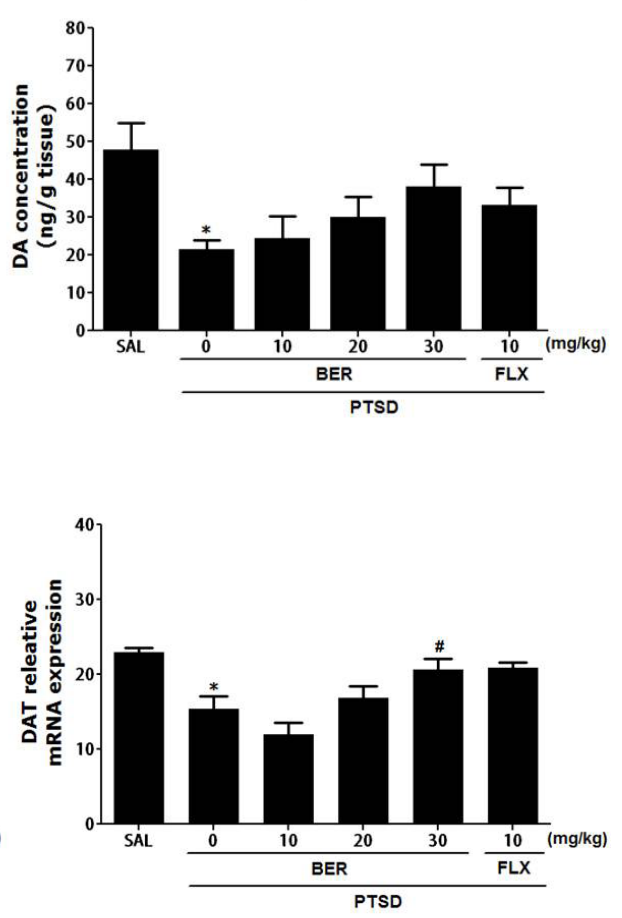

Fig. 5. Effects of BER administration on expression of tyrosine hydroxylase (TH), DA transporter (DAT), and vesicular monoamine transporter-2 (VMAT2) mRNA in rats during SPS-induced anxiety symptoms. Polymerase chain reaction bands on agarose gel and their relative intensities are indicated. The expression levels of TH, DAT, and VMAT-2 mRNA were normalized to that of glyceraldehyde 3-phosphate dehydrogenase (GAPDH) mRNA as an internal control. ${ }^{*} p<0.05,{ }^{* *} p<0.01$ vs. the SAL group, ${ }^{\#} p<0.05,{ }^{*} p<0.01$ vs. the PTSD group. of rats by $34.12 \%$ compared with rats in the saline-treated group $(p<0.01)$. However, administration of BER inhibited the SPSinduced decrease in DA levels in the medial prefrontal cortex, although this result was only marginally statistically significant. The ELISA results showed that SPS exposure for 14 days significantly decreased the DA concentration in the amygdala of rats by $45.03 \%$ compared with rats in the saline-treated group $(\mathrm{p}<0.05)$. However, the administration of BER inhibited the SPS-induced decrease in DA levels in the amygdala; this result was only marginally significant.

\section{Effects of BER on expression of TH, DAT, and VMAT-2 mRNA in the hippocampus following SPS}

RC-PCR was conducted to examine the effect of BER on the expression levels of TH, DAT, and VMAT-2 mRNA in the hip- 
pocampus of rats exposed to SPS (Fig. 5). The mRNA level of TH in the PTSD group decreased significantly compared with that in the SAL group $(p<0.01)$. The decreased expression levels of TH mRNA in the SPS group were significantly re-established to levels similar to those in the SAL group after administration of $30 \mathrm{mg} / \mathrm{kg}$ BER $(\mathrm{p}<0.05)$. DAT and VMAT-2 mRNA levels in the PTSD group were decreased significantly compared with those in the SAL group $(\mathrm{p}<0.05)$. The decreased expression levels of DAT mRNA in the SPS group was significantly re-established to a level similar to that seen in the SAL group after receiving 30 $\mathrm{mg} / \mathrm{kg}$ BER $(\mathrm{p}<0.05)$. The decreased expression level of VMAT2 mRNA in the SPS group was significantly re-established by 30 $\mathrm{mg} / \mathrm{kg}$ BER, although this result was only marginally significant. These results indicate that expression of TH and DAT mRNA in the hippocampus of rats receiving $30 \mathrm{mg} / \mathrm{kg}$ of BER was similar to that of rats receiving $10 \mathrm{mg} / \mathrm{kg}$ FLX.

\section{DISCUSSION}

Administering BER after SPS exposure significantly increased the time spent in the open arms in the EPM, reduced the anxiety index, and decreased grooming behavior. Administering BER after SPS also significantly reduced anxiety-like behaviors, as indicated by an increase in the time spent in the central zone and the number of central zone crossings during the OFT. BER also restored the moving distance and rearing frequency in the OFT. Thus, BER appeared to act as an anxiolytic by preventing reductions in brain levels of DA. Therefore, these results could lead to the development of novel therapeutics for treating PTSD.

Prior behavioral investigations indicate that SPS is a relevant rodent model of PTSD [1]. The behavioral features induced by SPS include cognitive dysfunction [31,32], disrupted extinction learning [33,34], exaggerated startle responses [1], and heightened fear [35], which are relevant to the clinical symptoms of intrusion, avoidance, and hyperarousal seen in patients with PTSD [1]. Therefore, our study utilized the SPS to assess anxiety-like behavioral effects. Our behavioral findings are consistent with the clinical relationship between traumatic stress and anxiety (data not shown).

Stressful life events are also a risk factor for major depression or anxiety, and DAergic dysfunction is apparent in individuals with these disorders [14]. The SPS procedure as a rodent model of PTSD decreased the proportion of time that rats spent and the number of entries into the open arms on the EPM. The results of our behavioral investigation demonstrated the anxiolytic-like effects of BER in an animal model of anxiety. Administering BER after SPS significantly reduced anxiety-like behaviors on the EPM test, as indicated by exploratory behaviors, and more entries into the open arms [36]. Administering BER after SPS also significantly increased the number of central zone crossings on the OFT [29]. These changes in anxiety-like behaviors appeared to be specific and not locomotor activity impairment because the length of the OFT and EPM test track was similar for all groups. In this study, the grooming behavior data also revealed that rats treated with BER adapted better to novel stress, consistent with previous EPM test results [37]. Therefore, because behaviors on the EPM test and OFT are related to DAergic system-associated psychological symptoms, our results indicate that BER may inhibit DAergic system dysregulation.

Our results show that anxiety following SPS is consistent with an impaired DAergic system in patients with PTSD. They also show reduced tissue content of DA in the medial prefrontal cortex, hippocampus, striatum, and amygdala of SPS rats, thereby indicating a reduction in DAergic tone in the mesolimbic pathway. Therefore, PTSD symptoms are re-experienced due to dysfunction or imbalance of DA within the anxiety circuit areas of the medial prefrontal cortex, hippocampus, striatum, and amygdala [38]. Neurobiologic events leading to dysregulation of the DA system after SPS are supported by some studies of severe stressors, such as life events [1,39]. Disruption of anxiety-related circuitry or alterations in the stress-reactivating system following SPS in the medial prefrontal cortex, hippocampus, striatum, and amygdala may be related to dysfunction in other brain regions in patients with PTSD [38]. At least one study thoroughly reviewed the effects of SPS on these structures and their role in PTSD neuropathology [38]. Dysregulation of mesolimbic DA function in the SPS model might include the contribution of glutamate, norepinephrine, glucocorticoid, or corticotropin-releasing factor, and the serotonin systems in the rat [40]. Therefore, DAergic dysfunction is hypothesized to contribute to anxiety-like behavior [38].

We hypothesize that BER modulation of SPS-induced anxietylike symptoms is associated with impaired functioning of central DA. We have shown that animals with PTSD treated with BER had significantly increased DA levels in the hippocampus and striatum, and that this may have inhibited the pathophysiology of PTSD. The effects of BER can be reversed by manipulating DA [38]. Thus, our results indicate that BER, like FLX, restores the behavior and neurochemical alterations associated with anxietylike symptoms by modulating the DA system in the brain [41].

The present study demonstrated concomitant changes of neural transmission in candidate catecholamines, in which DA efflux in the hippocampus was reduced after 2 weeks in rats that underwent the SPS procedure. We then observed a more dynamic relationship in which an area-dependent consistency existed between the release and reuptake of specific DA after a traumatic experience. The SPS procedure reduced DA efflux and TH, DAT, and VMAT-2 mRNA levels in the hippocampus, indicating possible involvement of presynaptic adaptation or plasticity [42]. TH, synthesizes DA and is an important player in traumatic stressinduced activation in the DAergic system and in stress-related psychopathological conditions, including fear or anxiety [2]. Our results show that DA efflux and DAT decreased in the hippocampus, with a accompanying change in a marker of presynaptic 
DA activity, VMAT-2, in rats that underwent the SPS procedure [42]. For example, the SPS-altered efflux levels of DA also reflect neuronal adaptation in synaptic release rather than reuptake, because the concomitant reduction of VMAT-2 represents a reduced amount of the neurotransmitter in the vesicle available for exocytosis [43]. The concomitant deduction of both DA efflux and VMAT-2 in the hippocampus suggests that SPS may downregulate synaptic release of DA [42]. Therefore, we deduced that SPS decreased the expression levels of TH and DAT mRNA in the hippocampus as well as anxiety-like behaviors. BER restored hippocampal TH and DAT mRNA expression levels, suggesting that modulation of the DA system plays a role in the anxiolytic effects of BER. Thus, BER acted like an anxiolytic, possibly by modifying the DA system.

Meanwhile, studies on the effects of BER on DA have reported controversial results $[22,44]$. Some studies suggest that treatment with BER enhanced motor balance and coordination by preventing dopaminergic neuronal damage [44]. BER pretreatment at a dose of $50 \mathrm{mg} / \mathrm{kg}$ could prevent the reduction of TH immunoreactivity in the dopaminergic neurons [45]. In contrast, protoberberine alkaloids show inhibitory effect on DA biosynthesis by reducing tyrosine hydroxylase activity and $\mathrm{TH}$ gene expression in PC12 cell [22]. BER has been able to protect human dopaminergic neuronal cells against 6-hydroxydopamine (6-OHDA) neurotoxicity through the induction of heme oxygenase-1 [46]. These results suggest that BER have a dual action, depending upon central nervous and peripheral systems, and that the functions of BER are closely relate to catecholamine content [47]. These results suggest that BER exhibits dual functions, which are dependent on the experimental design, including the concentrations and treatment periods used. TH is the first and rate-limiting step in DA production. Inhibition of TH would be expect to lower DA synthesis. Therefore, we asked whether BER increases enzymes required for dopamine synthesis. We found that BER enhanced basal TH activity. Our results have shown that the neuroprotection effect of BER on anxiety-like behavior reflects the increase of dopaminergic biosynthesis or transmission. This suggestion is strongly strengthen by previous studies showing that treatment of BER increased DA biosynthesis in the brain [44].

Even though several studies have indicated that BER may have pharmacologic effects on the central DA system [47], this is the first demonstration of BER action on PTSD-induced behavioral and neurochemical activity. Little is currently know about the action of BER on the central DA system at cellular or molecular levels. Other kinds of protoberberines, the tetrahydroprotoberberines (THPBs), were previously show to display the highest affinity for the D1- and D2-like receptors [48]. Our preliminary data suggest that BER may bind to DA-receptor sites (unpublished observations). In addition to the clinical study of BER on anxiety symptoms, further pharmacologic actions of BER on the central dopaminergic systems should be investigate in the future.

Meanwhile, BER may have protective effects on several brain structures including the cerebral cortex and hippocampus, exerting beneficial effects on anxiety-like symptoms and DA activity. The multifaceted abilities of BER could be attribute to its strong neuroprotective effects. The preventative effect of BER treatment on maintaining increase DA level after SPS exposure suggests that BER may exert some effects on mechanisms that regulate anxiety-like symptoms control, particularly as BER can cross the blood-brain barrier where it may have a direct or indirect effect on the hippocampus. Some studies suggested that BER pretreatment at a dose of $50 \mathrm{mg} / \mathrm{kg}$ could significantly decrease apomorphine-induced rotations, attenuate loss of Nissl-stained substantianigra pars compacta (SNC) neurons and prevent the reduction of TH immunoreactivity in SNC dopaminergic neurons [45]. In contrast, post-lesion treatment of 6-OHDA-lesioned rats with BER did not show any improvement in apomorphineinduced rotations and was not capable of restoring dopamine level and TH level [22]. In that study, the restorative and not the neuroprotective potential of BER was evaluated and for this reason, BER was not capable to improve the condition when used after lesioning the nigrostriatal system. However, in our study, BER was administered as a neuroprotective agent during SPS exposure and for this reason, our obtained results are different compared with other studies as mentioned above. Based upon our results, it may best evidenced that BER could reduce dopaminergic neurodegeneration by SPS-induced damage.

Taken together, these results suggest that presynaptic DA is crucial after traumatic experiences and that DA hypofunction in the hippocampus appears useful for interpreting the long-term adaptation following a traumatic experience. Administering BER after SPS exposure significantly increased the time spent exploring the open arms on the EPM test, reduced the anxiety index, and decreased grooming behavior. Administering BER after SPS also significantly increased the time spent in the central zone and increased the number of central zone crossings during the OFT. Therefore, BER was associated with anxiolytic-like effects in the EPM test and OFT, possibly by modifying the DA system. These findings indicate that BER can ameliorate the neurochemical responses and psychological-related behaviors involved in anxiety. Thus, BER may be an alternative treatment for preventing anxiety-like behaviors associated with PTSD. These results could lead to the development of novel therapeutics for treating PTSD.

\section{ACKNOWLEDGEMENTS}

This research was supported by a Grant from the National Research Foundation of Korea funded by the Korean government (2016R1D1A1A09917012). 


\section{CONFLICTS OF INTEREST}

The authors declare no conflicts of interest.

\section{REFERENCES}

1. Enman NM, Arthur K, Ward SJ, Perrine SA, Unterwald EM. Anhedonia, reduced cocaine reward, and dopamine dysfunction in a rat model of posttraumatic stress disorder. Biol Psychiatry. 2015;78:871879.

2. Wilson CB, Ebenezer PJ, McLaughlin LD, Francis J. Predator exposure/psychosocial stress animal model of post-traumatic stress disorder modulates neurotransmitters in the rat hippocampus and prefrontal cortex. PLoS One. 2014;9:e89104.

3. Ji LL, Tong L, Xu BK, Fu CH, Shu W, Peng JB, Wang ZY. Intra-hippocampal administration of ZIP alleviates depressive and anxietylike responses in an animal model of posttraumatic stress disorder. Behav Brain Funct. 2014;10-28.

4. Rau V, DeCola JP, Fanselow MS. Stress-induced enhancement of fear learning: an animal model of posttraumatic stress disorder. Neurosci Biobehav Rev. 2005;29:1207-1223.

5. Shin LM, Liberzon I. The neurocircuitry of fear, stress, and anxiety disorders. Neuropsychopharmacology. 2010;35:169-191.

6. Hadad NA, Wu L, Hiller H, Krause EG, Schwendt M, Knackstedt LA. Conditioned stress prevents cue-primed cocaine reinstatement only in stress-responsive rats. Stress. 2016;19:406-418.

7. Lin CC, Tung CS, Lin PH, Huang CL, Liu YP. Traumatic stress causes distinctive effects on fear circuit catecholamines and the fear extinction profile in a rodent model of posttraumatic stress disorder. Eur Neuropsychopharmacol. 2016;26:1484-1495.

8. Pitman RK, Rasmusson AM, Koenen KC, Shin LM, Orr SP, Gilbertson MW, Milad MR, Liberzon I. Biological studies of post-traumatic stress disorder. Nat Rev Neurosci. 2012;13:769-787.

9. Yamamoto S, Morinobu S, Takei S, Fuchikami M, Matsuki A, Yamawaki S, Liberzon I. Single prolonged stress: toward an animal model of posttraumatic stress disorder. Depress Anxiety. 2009;26:11101117.

10. Eskandarian S, Vafaei AA, Vaezi GH, Taherian F, Kashefi A, Rashidy-Pour A. Effects of systemic administration of oxytocin on contextual fear extinction in a rat model of post-traumatic stress disorder. Basic Clin Neurosci. 2013;4:315-322.

11. Kohda K, Harada K, Kato K, Hoshino A, Motohashi J, Yamaji T, Morinobu S, Matsuoka N, Kato N. Glucocorticoid receptor activation is involved in producing abnormal phenotypes of single-prolonged stress rats: a putative post-traumatic stress disorder model. Neuroscience. 2007;148:22-33.

12. Pitman RK. Overview of biological themes in PTSD. Ann N Y Acad Sci. 1997;821:1-9.

13. Shea A, Walsh C, Macmillan H, Steiner M. Child maltreatment and HPA axis dysregulation: relationship to major depressive disorder and post traumatic stress disorder in females. Psychoneuroendocrinology. 2005;30:162-178.

14. Lee B, Sur B, Yeom M, Shim I, Lee H, Hahm DH. L-tetrahydropalmatine ameliorates development of anxiety and depression-related symptoms induced by single prolonged stress in rats. Biomol Ther
(Seoul). 2014;22:213-222.

15. Gemmel M, Rayen I, van Donkelaar E, Loftus T, Steinbusch HW, Kokras N, Dalla C, Pawluski JL. Gestational stress and fluoxetine treatment differentially affect plasticity, methylation and serotonin levels in the PFC and hippocampus of rat dams. Neuroscience. 2016; 327:32-43

16. Garabadu D, Ahmad A, Krishnamurthy S. Risperidone attenuates modified stress-re-stress paradigm-induced mitochondrial dysfunction and apoptosis in rats exhibiting post-traumatic stress disorderlike symptoms. J Mol Neurosci. 2015;56:299-312.

17. Liberzon I, López JF, Flagel SB, Vázquez DM, Young EA. Differential regulation of hippocampal glucocorticoid receptors mRNA and fast feedback: relevance to post-traumatic stress disorder. J Neuroendocrinol. 1999;11:11-17.

18. Nie H, Peng Z, Lao N, Wang H, Chen Y, Fang Z, Hou W, Gao F, Li X, Xiong L, Tan Q. Rosmarinic acid ameliorates PTSD-like symptoms in a rat model and promotes cell proliferation in the hippocampus. Prog Neuropsychopharmacol Biol Psychiatry. 2014;51:16-22.

19. Lee B, Sur B, Yeom M, Shim I, Lee H, Hahm DH. Effect of berberine on depression- and anxiety-like behaviors and activation of the noradrenergic system induced by development of morphine dependence in rats. Korean J Physiol Pharmacol. 2012;16:379-386.

20. Hwang YH, Cho WK, Jang D, Ha JH, Jung K, Yun HI, Ma JY. Effects of berberine and hwangryunhaedok-tang on oral bioavailability and pharmacokinetics of ciprofloxacin in rats. Evid Based Complement Alternat Med. 2012;2012:673132.

21. Moghaddam HK, Baluchnejadmojarad T, Roghani M, Khaksari M, Norouzi P, Ahooie M, Mahboobi F. Berberine ameliorate oxidative stress and astrogliosis in the hippocampus of STZ-induced diabetic rats. Mol Neurobiol. 2014;49:820-826.

22. Kwon IH, Choi HS, Shin KS, Lee BK, Lee CK, Hwang BY, Lim SC, Lee MK. Effects of berberine on 6-hydroxydopamine-induced neurotoxicity in PC12 cells and a rat model of Parkinson's disease. Neurosci Lett. 2010;486:29-33.

23. Qin-Wei Z, Yong-Guang LI. Berberine attenuates myocardial ischemia reperfusion injury by suppressing the activation of PI3K/AKT signaling. Exp Ther Med. 2016;11:978-984.

24. Zhu F, Qian C. Berberine chloride can ameliorate the spatial memory impairment and increase the expression of interleukin-1beta and inducible nitric oxide synthase in the rat model of Alzheimer's disease. BMC Neurosci. 2006;78-87.

25. Mantsch JR, Li SJ, Risinger R, Awad S, Katz E, Baker DA, Yang Z. Levo-tetrahydropalmatine attenuates cocaine self-administration and cocaine-induced reinstatement in rats. Psychopharmacology (Berl). 2007;192:581-591.

26. Yun J. L-tetrahydropalmatine inhibits methamphetamine-induced locomotor activity via regulation of 5-HT neuronal activity and dopamine D3 receptor expression. Phytomedicine. 2014;21:1287-1291.

27. Peng WH, Wu CR, Chen CS, Chen CF, Leu ZC, Hsieh MT. Anxiolytic effect of berberine on exploratory activity of the mouse in two experimental anxiety models: interaction with drugs acting at 5-HT receptors. Life Sci. 2004;75:2451-2462.

28. Patki G, Li L, Allam F, Solanki N, Dao AT, Alkadhi K, Salim S. Moderate treadmill exercise rescues anxiety and depression-like behavior as well as memory impairment in a rat model of posttraumatic stress disorder. Physiol Behav. 2014;130:47-53.

29. Lee B, Sur B, Cho SG, Yeom M, Shim I, Lee H, Hahm DH. Gin- 
senoside $\mathrm{Rb} 1$ rescues anxiety-like responses in a rat model of posttraumatic stress disorder. J Nat Med. 2016;70:133-144.

30. Yeom M, Sur BJ, Park J, Cho SG, Lee B, Kim ST, Kim KS, Lee H, Hahm DH. Oral administration of Lactobacillus casei variety rhamnosus partially alleviates TMA-induced atopic dermatitis in mice through improving intestinal microbiota. J Appl Microbiol. 2015;119:560-570.

31. Eagle AL, Fitzpatrick CJ, Perrine SA. Single prolonged stress impairs social and object novelty recognition in rats. Behav Brain Res. 2013;256:591-597.

32. Peng Y, Feng SF, Wang Q, Wang HN, Hou WG, Xiong L, Luo ZJ, Tan QR. Hyperbaric oxygen preconditioning ameliorates anxietylike behavior and cognitive impairments via upregulation of thioredoxin reductases in stressed rats. Prog Neuropsychopharmacol Biol Psychiatry. 2010;34:1018-1025.

33. Knox D, George SA, Fitzpatrick CJ, Rabinak CA, Maren S, Liberzon I. Single prolonged stress disrupts retention of extinguished fear in rats. Learn Mem. 2012;19:43-49.

34. Yamamoto S, Morinobu S, Fuchikami M, Kurata A, Kozuru T, Yamawaki S. Effects of single prolonged stress and D-cycloserine on contextual fear extinction and hippocampal NMDA receptor expression in a rat model of PTSD. Neuropsychopharmacology. 2008;33:2108-2116.

35. Khan S, Liberzon I. Topiramate attenuates exaggerated acoustic startle in an animal model of PTSD. Psychopharmacology (Berl). 2004;172:225-229.

36. Cohen H, Liu T, Kozlovsky N, Kaplan Z, Zohar J, Mathé AA. The neuropeptide Y (NPY)-ergic system is associated with behavioral resilience to stress exposure in an animal model of post-traumatic stress disorder. Neuropsychopharmacology. 2012;37:350-363.

37. Serova LI, Laukova M, Alaluf LG, Sabban EL. Intranasal infusion of melanocortin receptor four (MC4R) antagonist to rats ameliorates development of depression and anxiety related symptoms induced by single prolonged stress. Behav Brain Res. 2013;250:139-147.

38. Lin CC, Tung CS, Liu YP. Escitalopram reversed the traumatic stress-induced depressed and anxiety-like symptoms but not the deficits of fear memory. Psychopharmacology (Berl). 2016;233:11351146.

39. Zhang XG, Zhang H, Liang XL, Liu Q, Wang HY, Cao B, Cao J, Liu S, Long YJ, Xie WY, Peng DZ. Epigenetic mechanism of maternal post-traumatic stress disorder in delayed rat offspring development: dysregulation of methylation and gene expression. Genet Mol Res. 2016;15:15039009.

40. Wang HT, Han F, Shi YX. Activity of the 5-HT1A receptor is involved in the alteration of glucocorticoid receptor in hippocampus and corticotropin-releasing factor in hypothalamus in SPS rats. Int JMol Med. 2009;24:227-231.

41. Aykaç A, Aydın B, Cabadak H, Gören MZ. The change in muscarinic receptor subtypes in different brain regions of rats treated with fluoxetine or propranolol in a model of post-traumatic stress disorder. Behav Brain Res. 2012;232:124-129.

42. Jichao S, Xinmin H, Xianguo R, Dongqi Y, Rongyi Z, Shuang L, Yue Y, Yuchen S, Jingnan Y. Saikosaponin a alleviates symptoms of attention deficit hyperactivity disorder through downregulation of DAT and enhancing BDNF expression in spontaneous hypertensive rats. Evid Based Complement Alternat Med. 2017;2017:2695903.

43. Nikolaus S, Antke C, Beu M, Müller HW. Cortical GABA, striatal dopamine and midbrain serotonin as the key players in compulsive and anxiety disorders-results from in vivo imaging studies. Rev Neurosci. 2010;21:119-139.

44. Kim M, Cho KH, Shin MS, Lee JM, Cho HS, Kim CJ, Shin DH, Yang HJ. Berberine prevents nigrostriatal dopaminergic neuronal loss and suppresses hippocampal apoptosis in mice with Parkinson's disease. Int J Mol Med. 2014;33:870-878.

45. Negahdar F, Mehdizadeh M, Joghataei MT, Roghani M, Mehraeen F, Poorghayoomi E. Berberine chloride pretreatment exhibits neuroprotective effect against 6-hydroxydopamine-induced neuronal insult in rat. Iran J Pharm Res. 2015;14:1145-1152.

46. Bae J, Lee D, Kim YK, Gil M, Lee JY, Lee KJ. Berberine protects 6-hydroxydopamine-induced human dopaminergic neuronal cell death through the induction of heme oxygenase-1. Mol Cells. 2013;35:151-157.

47. Shin JS, Kim EI, Kai M, Lee MK. Inhibition of dopamine biosynthesis by protoberberine alkaloids in PC12 cells. Neurochem Res. 2000;25:363-368.

48. Sun H, Zhu L, Yang H, Qian W, Guo L, Zhou S, Gao B, Li Z, Zhou Y, Jiang H, Chen K, Zhen X, Liu H. Asymmetric total synthesis and identification of tetrahydroprotoberberine derivatives as new antipsychotic agents possessing a dopamine $\mathrm{D}_{1}, \mathrm{D}_{2}$ and serotonin 5-HT $\mathrm{H}_{1 \mathrm{~A}}$ multi-action profile. Bioorg Med Chem. 2013;21:856-868. 